\title{
Clinical Profile of Asymmetric Tonsillar Enlargement
}

\author{
Authors
}

\section{Parvathy A $\mathbf{P}^{1}$, Sindhu V Nath ${ }^{2}$, Satheesh Somaraj ${ }^{3}$, Jayaprabha Sathyabhama ${ }^{4}$, Venugopal $\mathbf{M}^{5}$}

${ }^{1}$ Junior Resident, Department of Otorhinolaryngology, Govt. Medical College, Thiruvananthapuram, Kerala, India

${ }^{2}$ Additional Professor, Department of Otorhinolaryngology, Govt. Medical College, Thiruvananthapuram, Kerala, India

${ }^{3}$ Professor and Head, Department of Otorhinolaryngology, Govt. Medical College, Thiruvananthapuram, Kerala, India

${ }^{4}$ Additional Professor Department of Otorhinolaryngology, Govt. Medical College, Thiruvananthapuram, Kerala, India

${ }^{5}$ Professor Department of Otorhinolaryngology, Govt. Medical College, Thiruvananthapuram, Kerala, India Corresponding Author

\section{Dr Sindhu V Nath}

Additional Professor, Department of Otorhinolaryngology, Govt. Medical College, Thiruvananthapuram, Kerala, India

\section{Abstract}

Objective: Primary- To study the aetiology of asymmetric tonsillar enlargement.

Materials and Methods: This study was conducted over a period of 18 months (January 2017 June 2018) and comprised of 54 patients of all age groups and either sex diagnosed as having asymmetric tonsillar enlargement and underwent either tonsillectomy or biopsy in the department of ENT, Government Medical College Hospital, Thiruvananthapuram. After obtaining consent for the study, detailed history taking and clinical examination were performed and other investigation reports were noted. Later the histopathology report of the operated specimen was obtained. All these results were analysed.

Results: Etiology was inferred from histopathological examination where 31(57.4\%) of them were diagnosed with chronic tonsillitis. This was followed by $26 \%$ of squamous cell carcinoma. There were 4 cases of tonsillar cyst and 2 cases of Large B cell Non-Hodgkin's lymphoma. There was one case each of hamartomatous polyp, tonsillolith and clear cell carcinoma. The benign lesions together constituted $68.5 \%$ (37 cases) and 31.5\% (17 cases) showed malignancy. Elderly males showed a significant association with the chance of developing malignancy.

Keyword: Asymmetric Tonsillar Enlargement.

\section{Introduction}

Tonsils are lymphoid tissue seen on either side of Oropharynx. They are primarily made of B lymphocytes and are lined by stratified squamous epithelium. Their main function is production of antibodies, gamma interferon and lymphokines. They are involved in the formation of immunoglobulins ( $\operatorname{IgA}, \operatorname{IgG}$ and $\operatorname{IgM})$, interferon and antistreptolysin. 
Unilateral tonsillar enlargement can be due to chronic infections (Tuberculosis, Syphilis, Actinomycoses), chronic inflammatory response (like sarcoidosis), benign tumours (papilloma, adenoma), other causes like tonsillar cyst, tonsilloliths, impacted tonsillar foreign body, intratonsillar abscess etc. or malignancy. The most common malignancy of tonsil is Squamous cell carcinoma which usually presents with ulceration of mucosa. Lymphoma usually appears as sub mucosal mass causing asymmetrical increase in size of tonsil. Unilateral tonsillar enlargement in children is considered as a dangerous sign of possibility of underlying lymphoma.

Tonsillectomy is associated with well documented short- and long-term risks. Considering the intraoperative and postoperative risks, including that of general anaesthesia, and its long-term effects on the normal development of the immune system, a clear rationale on the indications of surgery need to be defined. Various studies have been done to evaluate the modalities of treatment for unilateral tonsillar enlargement medical management and regular follow up or surgical management by biopsy and tonsillectomy.

\section{Aim}

Primary- To study the aetiology of asymmetric tonsillar enlargement.

Secondary- To assess the clinical features that are predictors of malignancy in a case of asymmetric tonsillar enlargement.

\section{Materials and Methods}

Study Design: Descriptive study

\section{Study Setting: Department of}

Otorhinolaryngology, Thiruvanathapuram, Kerala Government Medical College,

\section{Study Subjects}

All patients with asymmetric tonsillar enlargement presenting to the Department of Otorhinolaryngology, Government Medical College Hospital, Thiruvanathapuram during the study period constituted the study subjects. Study subjects will be selected according to inclusion and exclusion criterion. Both diffuse as well as localised swellings were included.

\section{Study Period}

18 months (January 2017 to June 2018).

\section{Sample Size}

No sampling was done. Minimum sample size was fixed as 50 .

\section{Inclusion Criterion}

All consecutive cases of asymmetric tonsillar enlargement, with an asymmetry of at least 1 grade. - Both diffuse as well as localised swellings will be taken up for the study.

\section{Exclusion Criteria}

- Patients not giving consent

- Peritonsillar abscess

- Parapharyngeal abscess and tumours

- Those who have undergone tonsillectomy

- Insignificant disparity less than 1 grade

- Acute tonsillitis

\section{Data Collection Tool}

- History and clinical findings

- Semi structured proforma

- Tonsillectomy or biopsy

- Histopathology report

\section{Method}

All clinically diagnosed cases of asymmetric tonsillar enlargement undergoing either biopsy or tonsillectomy in the ENT Department during the study period were identified. After obtaining consent for the study from the patient, detailed history was taken and clinical examinations performed.

Palatine tonsils were ranked according to Brodsky grading scale and its anatomical correlation

\begin{tabular}{|c|c|c|}
\hline GRADING & $\begin{array}{c}\text { PERCENTAGE OF AIR } \\
\text { WAY OBSTRUCTION }\end{array}$ & $\begin{array}{c}\text { ANATOMICAL } \\
\text { GRADING }\end{array}$ \\
\hline 0 & 0 & $\begin{array}{c}\text { Tonsil hidden behind } \\
\text { pillars }\end{array}$ \\
\hline I & $<25 \%$ & $\begin{array}{c}\text { Visible over anterior } \\
\text { pillar }\end{array}$ \\
\hline II & $25-50 \%$ & $\begin{array}{c}\text { Visible over posterior } \\
\text { pillar }\end{array}$ \\
\hline III & $50-75 \%$ & $\begin{array}{c}\text { Almost to midline } \\
\text { lateral to uvula }\end{array}$ \\
\hline IV & $>75 \%$ & Reaching midline \\
\hline
\end{tabular}


A proforma was used to record information from the patient. Particulars about patient history were obtained by interview technique. Patients underwent either tonsillectomy or tissue biopsy and the specimens were sent for histopathological examination. The histopathological reports were collected. The results were analysed, studied and compared with similar studies.

\section{Statistical Analysis}

Gathered data was entered in MS excel and analysed using SPSS software version 16. Frequencies of the parameters collected were analysed and expressed graphically. $\mathrm{p}$ value was calculated for different parameters collected to establish their significance.

\section{Ethical considerations}

Ethical clearance was obtained from the Human Ethics Committee, Medical College, Thiruvananthapuram (IEC.No.03/07/2017/MCT) prior to the study. Written informed consent was obtained from all study participants. Confidentiality was maintained regarding data given by the participants.

\section{Budget and funding}

No financial burden occurred for study subjects. All expenses were met by principal investigator

\section{Results}

- Among the 54 patients studied, asymmetric tonsillar enlargement was more common in males (55.6\%)(fig: 1 ).

- Most of them were present in age group $<10$ years. The mean age of presentation was 33.4 years.

- Most of the patients presented with bilateral tonsillar enlargement with asymmetry, accounting for a cumulative $53.7 \%$. The most common presenting complaint was throat pain seen in $92.6 \%$, followed by foreign body sensation throat in $48.1 \%$.

- Etiology was inferred from histopathological examination where $31(57.4 \%)$ of them were diagnosed with chronic tonsillitis. This was followed by $26 \%$ of squamous cell carcinoma. There were 4 cases of tonsillar cyst and 2 cases of Large B cell Non-Hodgkin's lymphoma. There was one case each of hamartomatous polyp, tonsillolith and clear cell carcinoma. The benign lesions together constituted $68.5 \%$ (37 cases) and $31.5 \%$ (17 cases) showed malignancy.

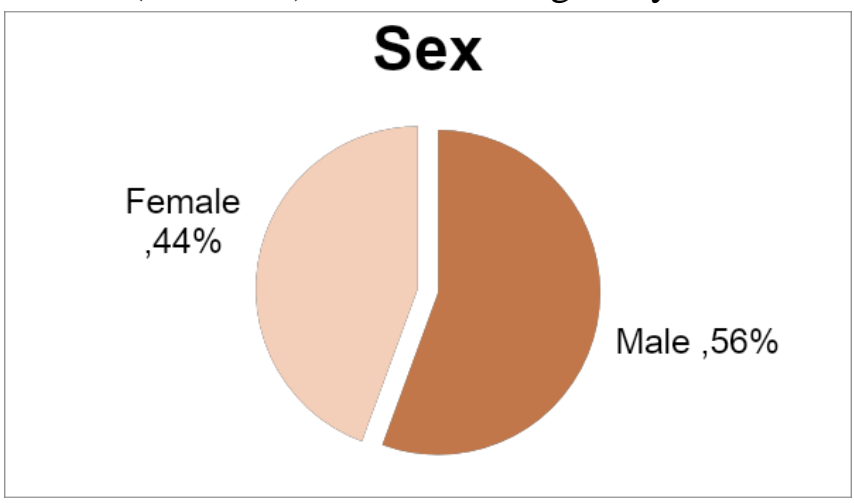

Fig 1

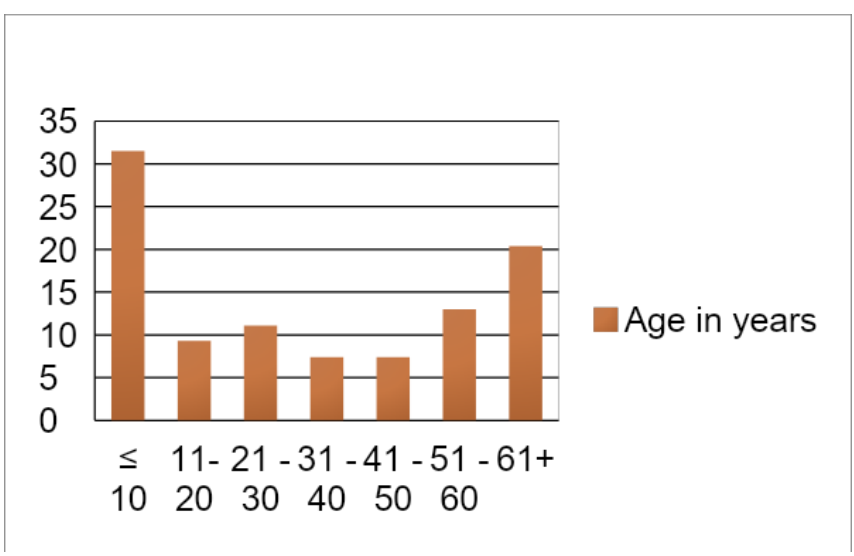

Fig: 2

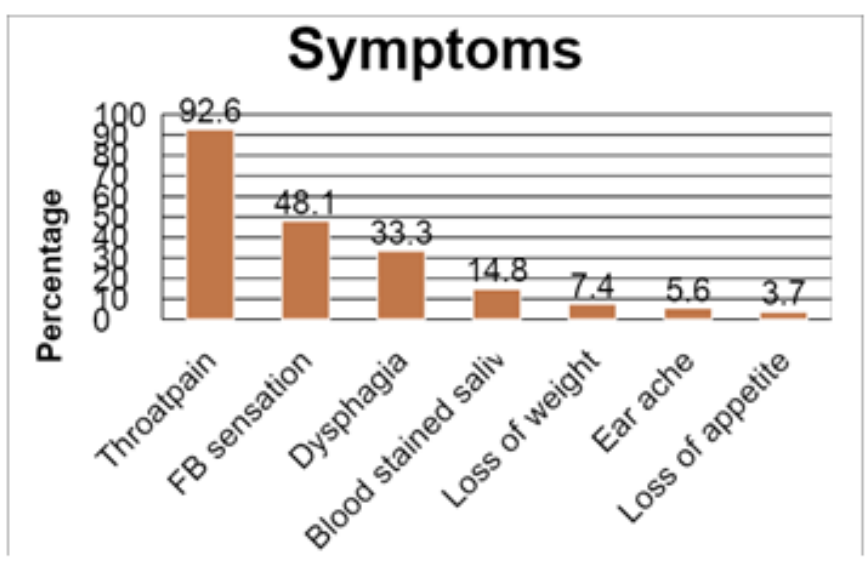

Fig 3 
Table: 1

\begin{tabular}{|l|c|c|}
\hline Symptoms & Frequency & Percent \\
\hline Throat pain & 50 & 92.6 \\
\hline Dysphagia & 18 & 33.3 \\
\hline Blood stained saliva & 8 & 14.8 \\
\hline FB sensation & 26 & 48.1 \\
\hline Ear ache & 3 & 5.6 \\
\hline Loss of weight & 4 & 7.4 \\
\hline Loss of appetite & 2 & 3.7 \\
\hline
\end{tabular}

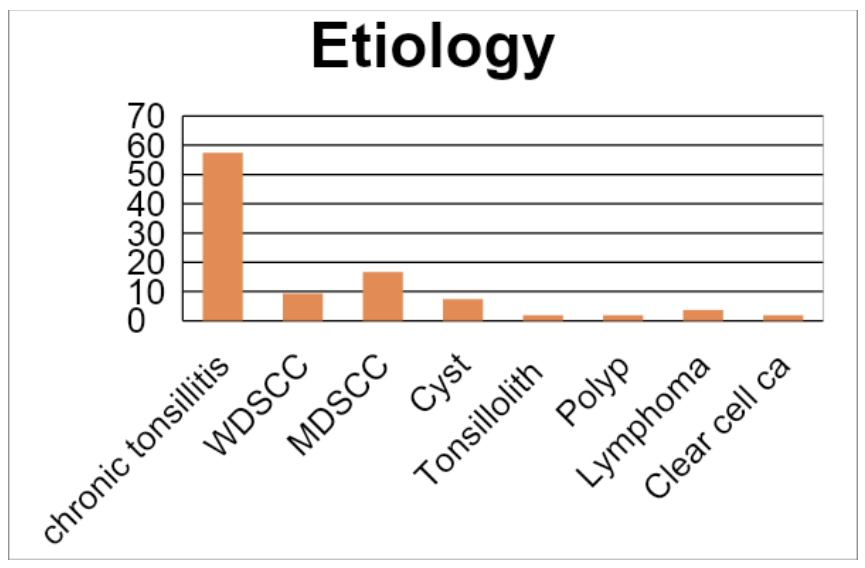

Fig: 4

Table: 2

\begin{tabular}{|l|l|l|}
\hline etiology & frequency & percentage \\
\hline Chronic tonsillitis & 39 & 57.4 \\
\hline Well differentiated SCC & 5 & 9.3 \\
\hline $\begin{array}{l}\text { Moderately } \\
\text { differentiated SCC }\end{array}$ & 9 & 16.7 \\
\hline Tonsillar cyst & 4 & 7.4 \\
\hline Tonsillar polyp & 1 & 1.9 \\
\hline Tonsillolith & 1 & 1.9 \\
\hline Lymphoma & 2 & 3.8 \\
\hline Clear cell carcinoma & 1 & 1.9 \\
\hline Total & 54 & 100 \\
\hline
\end{tabular}

\section{Discussion}

A diagnosis of asymmetric tonsillar enlargement was made after proper history elicitation and clinical examination. Asymmetry was diagnosed applying Brodsky's grading of the palatine tonsils. A difference of at least 1 grade was taken as asymmetry. After appropriate investigations, a tonsillar biopsy or a tonsillectomy was performed and the specimen was sent for histopathological examination. Histopathology report was collected.
Fifty four patients with at least one grade of palatine tonsillar asymmetry who underwent either tonsillectomy or tonsillar biopsy were included in this study. Among them, 30 were males constituting 55.6\%. A similar male preponderance was noticed in the study conducted by Ballin et al $^{1}$ with $54 \%$ being males.

The mean age of presentation was 33.4 years and majority of the patients $(31.5 \%)$ were from the age group below 10 years. This was in accordance with the study by Sumita et $\mathrm{al}^{2}$ where the mean age was 32 years. This was followed by $20.4 \%$ falling in the age group above 60. Such a bimodal distribution was reported in a study by Sunkaraneni et $\mathrm{al}^{3}$. Age distribution showed a wide range, with the youngest being 2 years old and eldest 74 years, which was seen in all the previous studies reviewed.

The most common presenting complaint was throat pain seen in $92.6 \%$ followed by foreign body sensation throat in $48.1 \%$. Similarly in the study by Aringa et al $94.4 \%$ presented with recurrent throat pain as the chief complaintpatients $^{4,5,6}$ had history of dysphagia, 8 presented with history of blood stained saliva. 3 had ear ache at presentation. Loss of weight and loss of weight was present in 4 and 2 cases respectively.

Etiology was inferred from histopathological examination where $31(57.4 \%)$ of them showed lymphoid hyperplasia consistent with a diagnosis of chronic tonsillitis. This was followed by $26 \%$ (14 cases) of squamous cell carcinoma. There were 4 cases of tonsillar cyst and 2 cases of Large B cell Non-Hodgkin's lymphoma. There were one case each of hamartomatous polyp, tonsillolith and clear cell carcinoma. Thus the benign lesions together constituted $68.5 \%$ (37 cases) and $31.5 \%$ (17 cases) showed malignancy. The predominance of histology of chronic tonsillitis was in accordance with the studies by Sumita et $\mathrm{al}^{2}$, Ballin et $\mathrm{al}^{1}$ and Sunkaraneni ${ }^{3}$ et al. 
But in all these studies, benign lesions of palatine tonsils constituted more than $85 \%$.

The higher incidence of malignancy in this study may be because the study was conducted at a tertiary care center. Being a tertiary care center, the percentage of malignant lesions is expected to be higher than in the general population due to higher referral rate. Of the two cases of lymphoma, both were B cell Non-Hodgkin's lymphoma. Both were in males aged more than 45 years. And none of them had constitutional symptoms. Similar results were obtained in studies by Spinu et $\mathrm{al}^{7}$, Sunkaraneni et $\mathrm{al}^{3}$ and Reiter et $\mathrm{al}^{8}$. In the study by Reiter et $\mathrm{al}^{8}$ of the 31 patients studied, only 2 had lymphoma. There were no cases of lymphoma in children with asymmetric tonsils which was in accordance with the study by Berkowitz et $\mathrm{al}^{9}$ and Weibel et $\mathrm{al}^{10}$.

Bridget et al where elderly female more than 50 years of age presented with incidently noticed swelling ${ }^{11,12}$. Analysis of the risk factors of malignancy of the 30 males in the study group, 13 were found to have malignancy. Hence male gender showed a significant association with the chance of developing malignancy ( $p$ value 0.036). So was the case with age more than 50 years with a $\mathrm{p}$ value $<0.01$. Thus elderly males possessed a higher chance of developing malignancy. In study by Beaty et $\mathrm{al}^{13}$, the mean age of malignancy was found to be 50.4 with a significant $\mathrm{p}$ value of 0.00018 .

Symptoms like blood stained saliva ( $p$ value $<0.001)$, unexplained loss of weight $(p=0.008)$ and loss of appetite $(\mathrm{p}=0.03)$ were found to be predictive of malignancy. A very strong association was found between blood stained saliva and chance of malignancy. Of the 8 patients who had complaints of blood stained saliva, all of them turned out to be malignant. Also loss of weight and loss of appetite were present only in those with malignancy. These were in accordance with the risk factors proposed by Spinou et $\mathrm{al}^{7}$, Cinar et $\mathrm{al}^{14}$ and Oluwasanmi et $\mathrm{al}^{15}$. Presence of persistent pain in the throat was more indicative of malignancy. However, a history of recurrent sore throat was found to be associated with reduced risk of developing malignancy with a $\mathrm{p}$ value $<0.001$. Such a parameter was not found to be included in any of the previous studies reviewed

\section{Conclusion}

- Asymmetric tonsillar enlargement was more common in males with a bimodal distribution in age.

- The most common presenting complaint was throat pain followed by foreign body sensation throat.

- Commonest aetiology was chronic tonsillitis followed by squamous cell carcinoma

- The benign lesions together constituted the majority about two-third.

- Male gender showed a significant association with the chance of developing malignancy.

- Symptoms like blood stained saliva, unexplained loss of weight and loss of appetite were found to be predictive of malignancy.

\section{Reference}

1. Ballin A, Koerner H, Ballin C, Pereira R, Alcantara L, et al. Palatine Tonsils Asymmetry: 10 Years Experience of the Otorhinolaryngology Service of the Clinical Hospital of the Federal University of Parana. Intl. Arch. Otorhinolaryngol. 2011;15(1):67-71

2. Sumitha R, Mohamed H. Unilateral Tonsillar Enlargement - a case series. International Journal of Pharma and Bio Sciences 2013;4(4):(B) $472-477$

3. Sunkaraneni V, Jones S, Prasai A, Fish B. Is unilateral tonsillar enlargement alone an 
indication for tonsillectomy?. The Journal of Laryngology \& Otology. 2006; E(21)

4. Aringa ARD, Juares AJC, Melo C, Nardi JC, Kobari K, Filho R. Histological analysis of tonsillectomy and adenoidectomy specimens - January 2001 to May 2003. 2005; 71(1): 18-22

5. Ikram M, Khan MA, Ahmed M, Siddiqui T, Mian MY. The histopathology of routine tonsillectomy specimens: results of a study and review of literature. Ear Nose Throat J 2000; 79(11): 880-2

6. Mudra RK, Verma J, Gupta A, Koshta V, Rathore S. Tonsillar papilloma: a rare case. Inter J Med Sci Res Prac 2015; 2(3): 162-3

7. Spinou C, Kubba H, Konstantinidis I, Johnston A. Role of tonsillectomy in histology for adults with unilateral tonsillar enlargement. Br J Oral Maxillofac Surg. 2005;43:144-7.

8. Reiter ER, Randolph GW, Pilch BZ. Microscopic detection of occult malignancy in the adult tonsil. Otolaryngol Head Neck Surg. 1999;120:190-4

9. Berkowitz RG, Mahadevan M. Unilateral tonsillar enlargement and tonsillar lymphoma in children. Ann Otol Rhinol Laryngol. 1999;108:876-9.

10. Weibel E. Pathological findings of clinical value in tonsils and adenoids. Acta Otolaryngol (Stockh) 1965;60:331-338.

11. Danielea L, Nikolarakosb D, Keenanb J, Schaeferb N, Lama AK. Clear cell carcinoma, not otherwise specified/ hyalinising clear cellcarcinoma of the salivary gland: The current nomenclature, clinical/pathological characteristics and management. Critical Reviews in Oncology/Hematology. 2016; 102:55-64.
12. Burgess B, Ananthanarayanan V, Charous S. Hyalinizing Clear Cell Carcinoma of the Tonsil: A Case Report. Head and Neck Pathol 2017; 11:580-83

13. Beaty M, Funk G, Karnell LH, Graham S, McCulloch T, Hoffman $\mathrm{H}$, et al. Risk factors for malignancy in adult tonsils. Head and Neck. 1998;20:399-403

14. Cinar F. Significance of asymptomatic tonsil asymmetry. Otolaryngol Head Neck Surg. 2004;131:101-3.

15. Oluwasanmi A, Word A, Balwin D, Sipaul F. Malignancy in asymmetrical but otherwise normal palatine tonsils. Ear, Nose and Throat Journal. 2006;85:661-3. 